\title{
What Does the Eye Tell the Brain?: Development of a System for the Large-Scale Recording of Retinal Output Activity
}

\author{
A. M. Litke, N. Bezayiff, E. J. Chichilnisky, W. Cunningham, W. Dabrowski, A. A. Grillo, M. Grivich, P. Grybos, \\ P. Hottowy, S. Kachiguine, R. S. Kalmar, K. Mathieson, D. Petrusca, M. Rahman, and A. Sher
}

\begin{abstract}
A multielectrode array system has been developed to study how the retina processes and encodes visual images. This system can simultaneously record the extracellular electrical activity from hundreds of retinal output neurons as a dynamic visual image is focused on the input neurons. The retinal output signals detected can be correlated with the visual input to study the neural code used by the eye to send information about the visual world to the brain. The system consists of the following components: 1) a $32 \times 16$ rectangular array of 512 planar microelectrodes with a sensitive area of $1.7 \mathrm{~mm}^{2}$; the electrode spacing is $60 \mu \mathrm{m}$ and the electrode diameter is $5 \mu \mathrm{m}$ (hexagonal arrays with 519 electrodes are under development); 2) eight 64-channel custom-designed integrated circuits to platinize the electrodes and ac couple the signals; 3) eight 64-channel integrated circuits to amplify, band-pass filter, and analog multiplex the signals; 4) a data acquisition system; and 5) data processing software. This paper will describe the design of the system, the experimental and data analysis techniques, and some first results with live retina. The system is based on techniques and expertise acquired in the development of silicon microstrip detectors for high-energy physics experiments.
\end{abstract}

Index Terms-Analog integrated circuits, biomedical electrodes, image processing, neural networks, visual system.

\section{INTRODUCTION}

$\mathbf{T}$ HIS paper describes a project [1]-[3] aimed at understanding the language and processing employed by the eye to send information about the external visual world to the brain. It will discuss the system we have developed to record the electrical signals from hundreds of retinal output neurons, a scale that approaches the number of inputs employed in the

Manuscript received November 15, 2003; revised March 29, 2004. This work was supported in part by the National Science Foundation through Award PHY-0245104, the National Institutes of Health, the Polish State Committee for Scientific Research under Project 7 T11B 054 21, and a NATO Collaborative Linkage Grant.

A. M. Litke, N. Bezayiff, A. A. Grillo, M. Grivich, S. Kachiguine, D. Petrusca, and A. Sher are with the Santa Cruz Institute for Particle Physics, University of California, Santa Cruz, CA 95064 USA (e-mail: alan.litke@cern. ch; bezayiff@maxwell.ucsc.edu; grillo@scipp.ucsc.edu; mgrivich@scipp. ucsc.edu; sergei@scipp.ucsc.edu; dumitru.petrusca@cern.ch; sasha@sscipp. ucsc.edu).

E. J. Chichilnisky and R. S. Kalmar are with the Salk Institute, La Jolla, CA 92037 USA (e-mail: ej@ salk.edu; kalmar@ salk.edu).

W. Cunningham, K. Mathieson, and M. Rahman are with the University of Glasgow, G12 8QQ Glasgow, U.K. (e-mail: w.cunningham@physics.gla.ac.uk; k.mathieson@physics.gla.ac.uk; m.rahman@physics.gla.ac.uk).

W. Dabrowski, P. Grybos, and P. Hottowy are with the AGH University of Science and Technology, Faculty of Physics and Nuclear Techniques, 30-059 Kraków, Poland (e-mail: W.Dabrowski@ftj.agh.edu.pl; grybos@ftj.agh.edu.pl; hottowy@fatcat.ftj.agh.edu.pl).

Digital Object Identifier 10.1109/TNS.2004.832706 visual cortex for neural computations. It will also discuss some first results on retinal processing based on experiments with live retinas.

The cornea and lens of the eye focus light from the external visual world onto the retina. The retina is a thin tissue that lines the back of the eye and that converts the input dynamic image into a set of electrical signals (action potentials or "spikes") that travel up the optic nerve to the brain. The human retina has approximately 100 million light-sensitive pixels (the photoreceptors, both rods and cones) that cover an area of about 10 $\mathrm{cm}^{2}$, and approximately one million output neurons (the retinal ganglion cells, RGCs) that generate the spike trains. The $10^{6}$ output fibers (axons) of the ganglion cells form the optic nerve. To study how the retina processes and encodes dynamic visual images, we record the patterns of electrical activity generated by hundreds of RGCs in response to a movie focused on the photoreceptors. The technology employed is based on silicon microstrip detector techniques and expertise developed for experiments in high-energy physics. It is an example of the application of expertise in high-energy physics instrumentation to neurobiology.

\section{EXPERIMENTAL TECHNIQUE}

The experimental technique is based on the work of Meister et al. [4]. Live retina is placed in a chamber on top of a twodimensional (2-D) array of microscopic electrodes fabricated on a glass substrate (Fig. 1). The tissue is bathed continuously with a flow of oxygenated physiological saline solution that passes through the chamber. In this manner, the retina can be kept alive for up to $15 \mathrm{~h}$.

A movie displayed on a computer monitor is focused by a microscope lens on the photoreceptors. In response to this dynamic image, the RGCs generate a set of spikes that produce local electrical signals on the extracellular electrodes, relative to a reference electrode (a platinum wire) immersed in the saline bath. These signals are ac-coupled by an application specific integrated circuit (ASIC - the "Platchip"), amplified, bandpass-filtered, and analog multiplexed by a second ASIC (the "Neurochip"), and then digitized and recorded by the data acquisition system.

\section{ELECTRODE ARRAYS}

Fig. 2 shows a cross-sectional view of a retina placed on top of a multielectrode array. The electrodes and connecting traces 


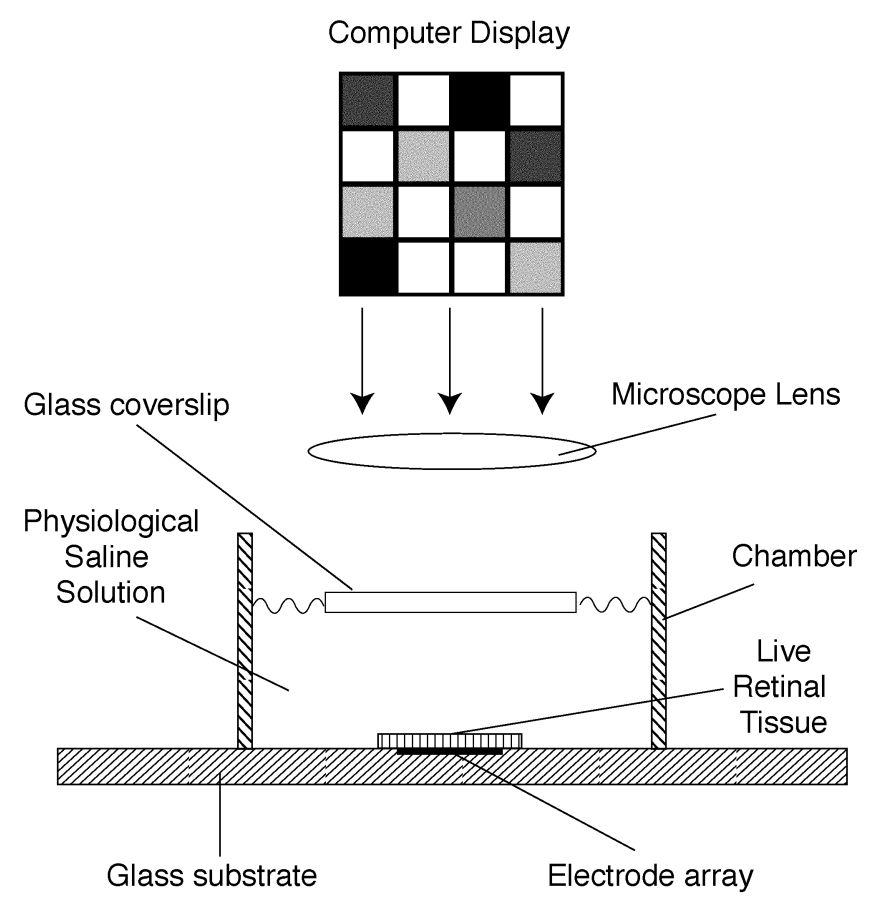

Fig. 1. Experimental setup.

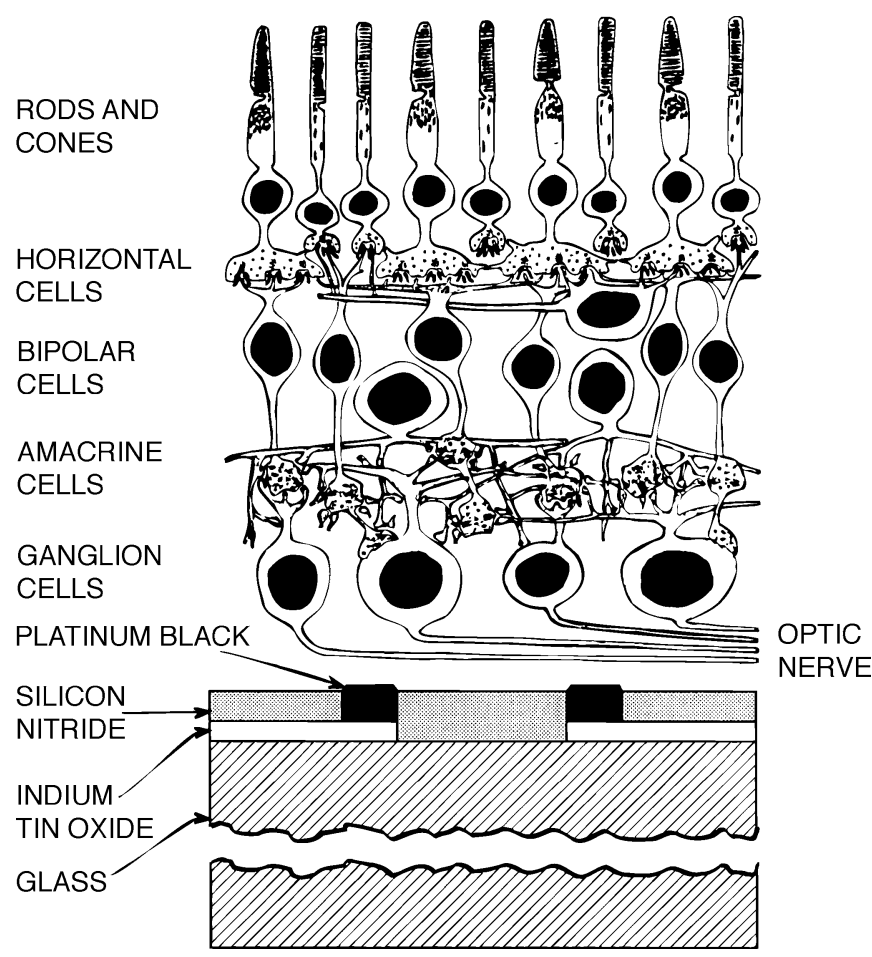

Fig. 2. Cross section of retina on top of a multielectrode array.

are fabricated from indium tin oxide (ITO) deposited on a glass substrate. The electrodes are formed from vias in an insulating layer of silicon nitride. Each electrode is electroplated with platinum to reduce the impedance of the electrode/saline solution interface. The electrode diameter is (typically) $5 \mu \mathrm{m}$.

Examples of electrode array geometries are shown in Fig. 3. For comparison, to provide a scale related to a neural computation, we show a 3.2-mm-diameter circle that represents the area on the retina surface that provides the input signals to a monkey

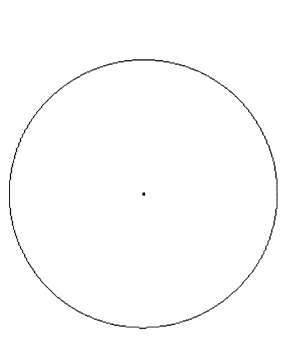

(a)

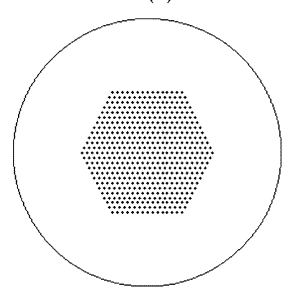

(d)

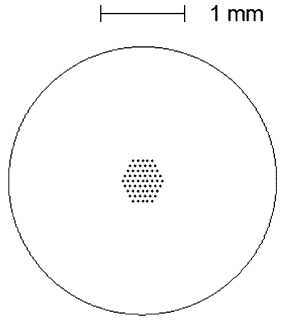

(b)

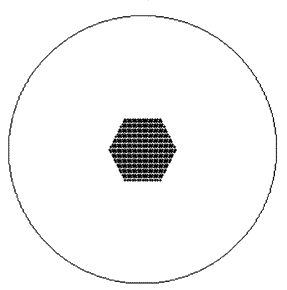

(e)

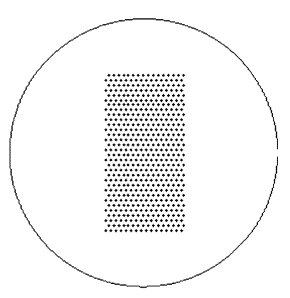

(c)

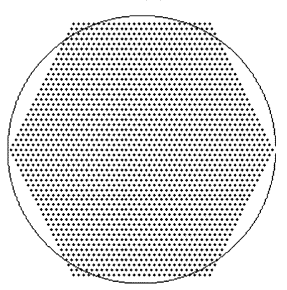

(f)
Fig. 3. Electrode array geometries. (a) Single electrode ("traditional"). (b) 61 electrodes (previous generation). (c) 512 electrodes (this paper). (d) 519 electrodes (fabrication underway). (e) 519 electrodes (high density with $30 \mu \mathrm{m}$ electrode spacing; under development). (f) 2053 electrodes (futuristic).

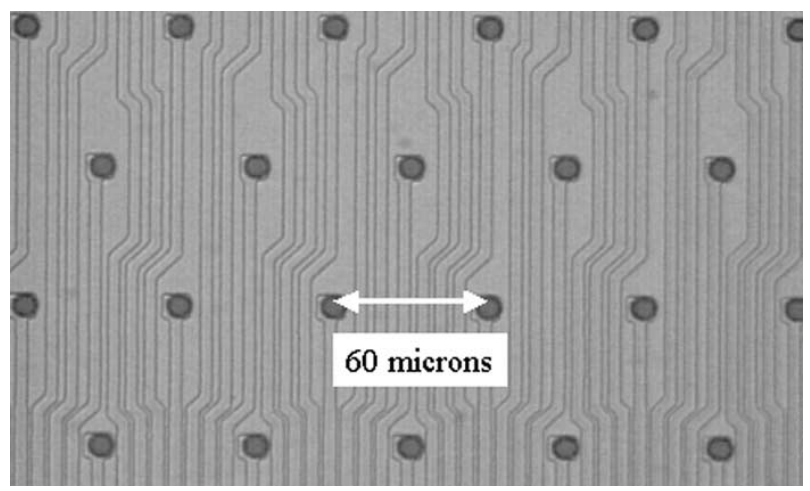

Fig. 4. Section of a 512-electrode array. The electrode spacing is $60 \mu \mathrm{m}$ and the electrode diameter is $5 \mu \mathrm{m}$.

middle temporal (MT) neuron-a member of a neuron class in the middle temporal region of the visual cortex. This class is thought to play a key role in the perception of motion.

Fig. 3(a) symbolically represents the traditional method used by neurobiologists to probe the retina: a single microelectrode records from a single neuron. For comparison, Fig. 3(b) displays an array that represents the state of the art as it was before the present study. It has 61 electrodes in a hexagonal geometry, 60 $-\mu \mathrm{m}$ electrode spacing, and a total area of $0.17 \mathrm{~mm}^{2}$. In a single monkey preparation, it can detect, typically, tens of neurons.

Fig. 3(c) shows the geometry of the array that is the main subject of this paper; it provides an order-of-magnitude improvement over the previous generation. It has 512 electrodes in a $32 \times 16$ rectangular geometry, $60-\mu$ m electrode spacing, and a total area of $1.7 \mathrm{~mm}^{2}$. It can detect hundreds of RGCs simultaneously in a single monkey retinal preparation, as discussed in Section VII. A photograph of a section of a 512-electrode array is shown in Fig. 4.

An hexagonal array [Fig. 3(d)] with 519 electrodes, 60- $\mu \mathrm{m}$ electrode spacing, and a total area of $1.7 \mathrm{~mm}^{2}$, is in fabrication. Fig. 3(e) illustrates a high-density hexagonal array that is under development. It will have 519 electrodes with an electrode 


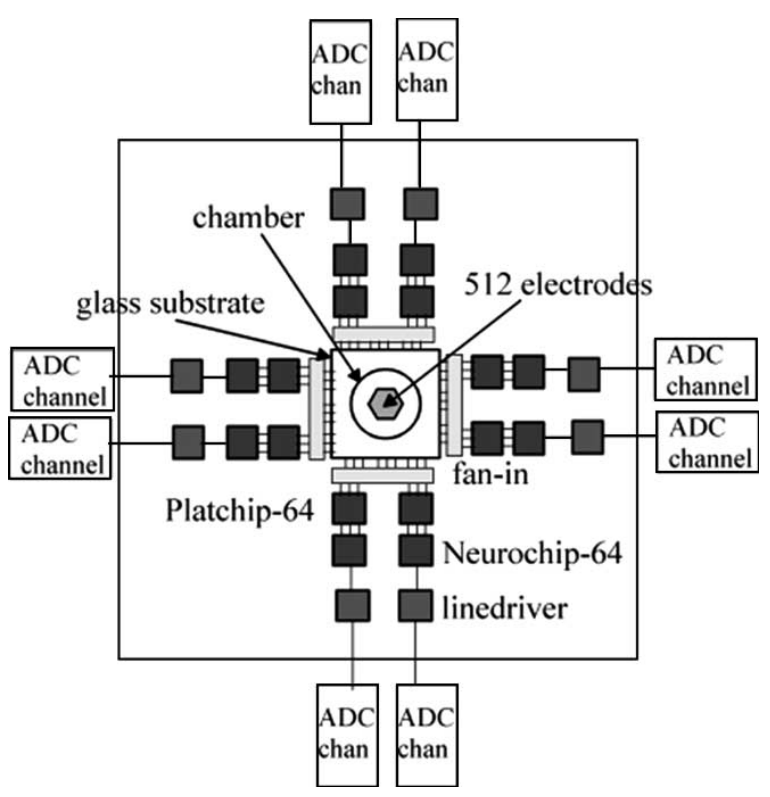

Fig. 5. Block diagram of the Neuroboard.

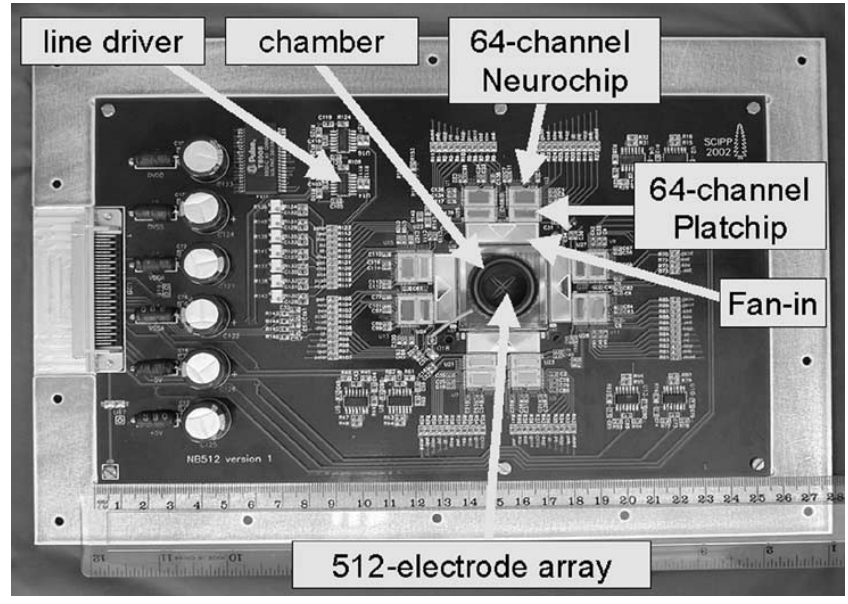

Fig. 6. Photograph of the Neuroboard.

spacing of $30 \mu \mathrm{m}$. Although it will have one quarter the area of the array shown in Fig. 3(d), it should be able to detect small neurons (such as the off-small neurons-see Section VII) with higher efficiency. Fig. 3(f) shows a futuristic array with 2053 electrodes, covering an area $\left(7.3 \mathrm{~mm}^{2}\right)$ well matched to the MT neuron input region. Details of the fabrication of the arrays are provided in [5].

\section{Readout System AND Data AcQuisition}

The electrode array is glued onto a printed circuit board (the "Neuroboard") for readout. A block diagram of the Neuroboard is shown in Fig. 5 and a photograph appears in Fig. 6. A magnified view of the central region of the Neuroboard is displayed in Fig. 7. The 512 electrodes are read out by a set of eight "Platchips" and eight "Neurochips."

Each Platchip (see Fig. 8) has 64 channels of 150-pF capacitors for ac-coupling the electrode signal to the corresponding Neurochip channel. Each Platchip channel also includes a current generator for electroplating each electrode with platinum

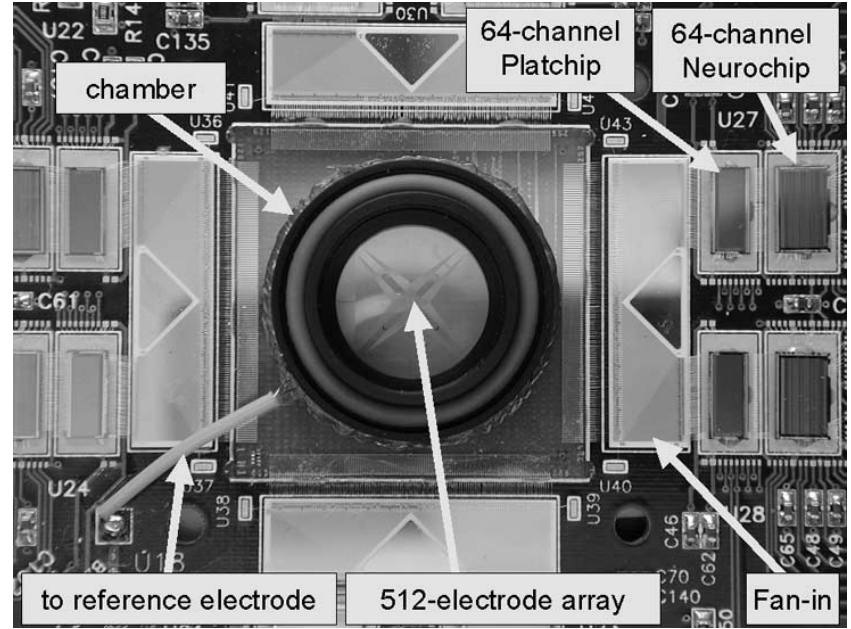

Fig. 7. Magnified view of the central region of the Neuroboard.

(0-1.2 $\mu \mathrm{A}$, controlled by a $5-\mathrm{b} \mathrm{DAC})$, and circuitry for providing a current pulse for neuron stimulation $(0-150 \mu \mathrm{A}$, controlled by an external analog signal with gain set by a 5-b DAC). The channel pitch is $120 \mu \mathrm{m}$ and the die size is $3.3 \times 7.8 \mathrm{~mm}^{2}$.

As shown in Fig. 9, each Neurochip has 64 channels of differential preamplification, bandpass filtering, output amplification, and a sample and hold, followed by a 64:1 analog multiplexer. The passband (which is tunable) is typically set to $80-2000 \mathrm{~Hz}$, the overall gain is typically 1500 , and the equivalent rms input noise is $\sim 5 \mu \mathrm{V}$. The channel pitch is $120 \mu \mathrm{m}$ and the die size is $4.8 \times 7.8 \mathrm{~mm}^{2}$. The Platchip and Neurochip are discussed in more detail in [6].

One note on signal/noise: at the system level, with an electrode array immersed in saline solution, the measured equivalent rms input noise is $\sim 7 \mu \mathrm{V}$. The typical signal amplitude threshold is $60 \mu \mathrm{V}$ and the signal amplitudes range up to $\sim 800 \mu \mathrm{V}$.

Data acquisition (DAQ) and recording is based on a personal computer (PC) running Labview [7]. This DAQ PC contains two ADC boards, each with four 12-b ADC channels [8]. Each of the eight multiplexed analog signals is digitized by an ADC channel at a rate of $1.28 \mathrm{MHz}$, corresponding to a sampling rate for each electrode channel of $20 \mathrm{kHz}$. A digital pattern generation board [9] is used to generate the control and synchronization signals for the Neurochips, the display computer, and the ADCs.

The DAQ computer records the data at a data rate of $15 \mathrm{MB} / \mathrm{s}$ on a hard disk. It also sends the data over a Gigabit/s Ethernet connection to a second PC, running Java, for data monitoring and diagnostics. A typical experiment, which may employ several pieces of retina, will last $\sim 24$ hours and generate close to one Terabyte of data.

\section{Data ANALYsis-First Steps}

The raw data recorded are the electrode signals, but the data of interest for the neurobiology are the spikes generated by individual neurons. Unfortunately, the electrode signals and the neuron signals are not in one-to-one correspondence. Each electrode picks up, in general, signals from several neurons, and each neuron, in general, produces signals on several electrodes. 


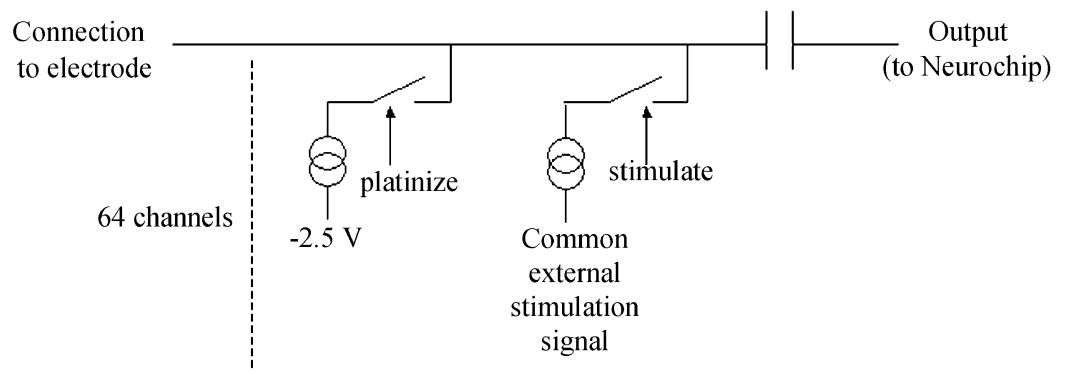

Fig. 8. Block diagram of the Platchip.

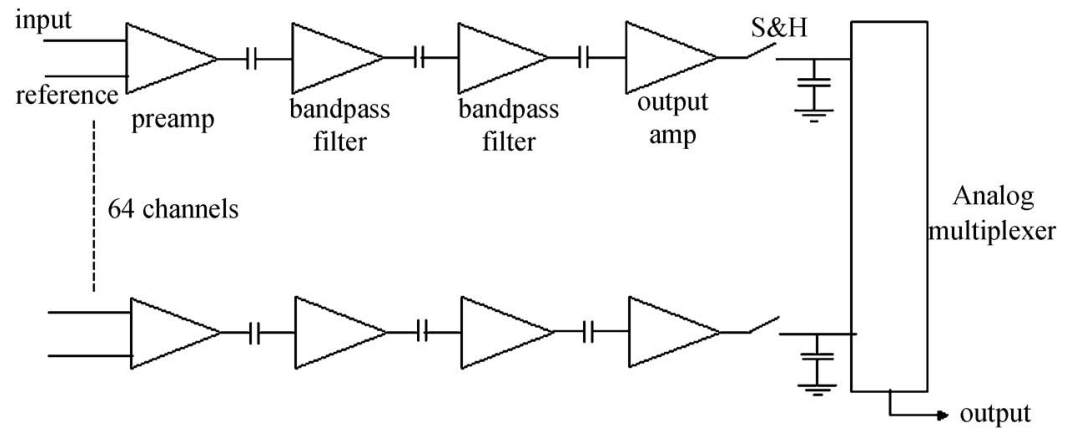

Fig. 9. Block diagram of the Neurochip.

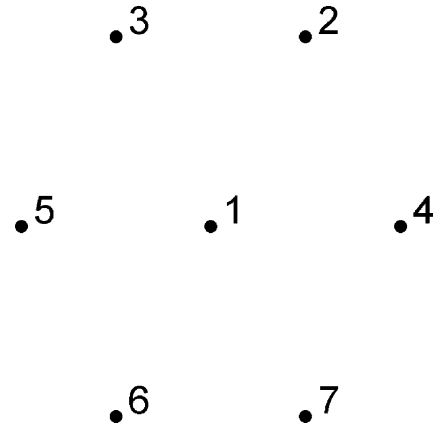

Fig. 10. Seed electrode (1), on which a spike has been identified, and its six nearest neighbors. The electrode spacing is $60 \mu \mathrm{m}$.

Thus, the first major stage in data analysis is to identify individual neurons from their characteristic spike signals.

As an initial step, a spike is identified as a signal amplitude on a single electrode that goes above, and then below, a preset threshold. As mentioned in Section IV, this threshold is typically $60 \mu \mathrm{V}$.

At this point, the traditional method to identify neurons is to manually look for clusters in a spike-amplitude versus spikewidth scatter plot, for spikes recorded on a single electrode [4]. However, the individual clusters are often obscured by the merging together of several such clusters from several neurons. Fortunately, the multielectrode array system reported here provides much more information than the single-electrode scatter plot: it records the analog waveforms on all the electrodes, preserving the spatial and temporal correlations associated with a spike on a "seed" electrode.

An important consideration concerning neuron identification by manual clustering is that it is a very time-consuming and subjective procedure. This approach is not very practical in a system with 512 electrodes. Therefore, our goal has been to develop automated neuron identification software that takes into account the multielectrode signal correlations. Moreover, with $\sim 1$ Terabyte of data to analyze after each experimental run, high data processing speed is necessary. With these factors in mind, we have developed custom software for automated identification of neurons, based on the following steps.

In the first step, for each identified spike on an electrode, a 182-dimensional vector is constructed from the analog waveforms on this electrode (1) and its six nearest neighbors (2-7; the electrode geometry is shown in Fig. 10). Each waveform consists of the 26 analog samples for the period $0.5 \mathrm{~ms}$ before the spike to $0.8 \mathrm{~ms}$ after the spike. Fifty superimposed traces of the combined waveforms are shown in Fig. 11.

In the second step, principal components analysis (PCA) is employed to reduce the dimensionality of the data by finding the $N$ most-significant variables (those with the highest variance), where these variables are linear combinations of the 182 measurements. In the example below, $N=5$, which is our typical value. Each spike, and its associated combined analog waveforms, are then represented as a point in an $N$-dimensional space. Fig. 12 shows a scatter plot of the two most significant variables for the data sample on which Fig. 11 is based.

In the third step, the spike waveform data, projected into this space of reduced dimensionality $N$, are searched for clusters that can be interpreted as arising from individual neurons. The Expectation Maximization algorithm is used to carry out the multidimensional clustering. In the example illustrated in Fig. 12, four clusters/neuron candidates are found, with the average signal waveforms shown in Fig. 13.

Some of the clusters may be due to not a single neuron but to a mixture of spikes from two or more neurons. In the fourth step, the autocorrelation function (ACF) is used to eliminate these 


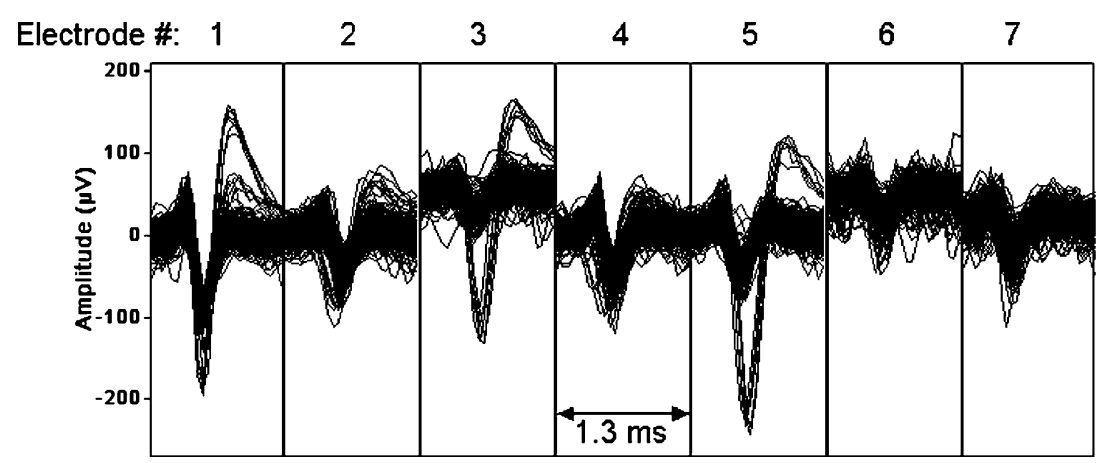

Fig. 11. Signal waveforms produced on the seed electrode (1) and its nearest neighbors (electrodes 2-7) when a spike is detected on electrode 1 . Fifty example traces are superimposed. One ADC count is equivalent to $0.7 \mu \mathrm{V}$ at the input.

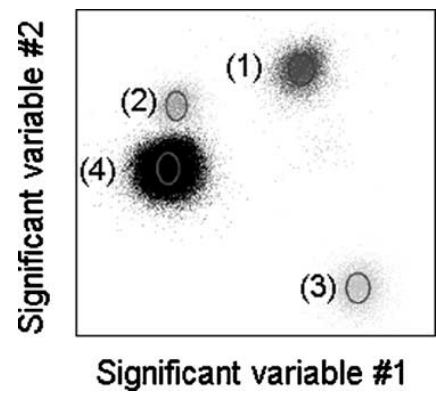

Fig. 12. Scatter plot of the two most significant variables, as found by PCA, for the spike waveform data. The data in five dimensions (not just the two displayed in this scatter plot) have been fit to a weighted sum of four five-dimensional Gaussians. These Gaussians are represented in this 2-D projection by the four ellipses.

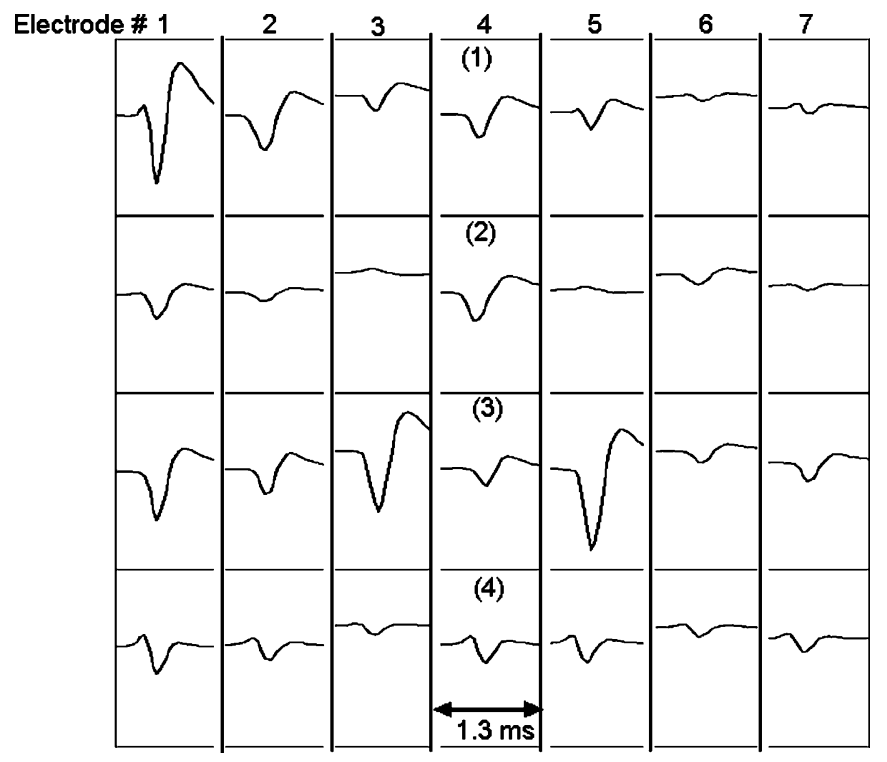

Fig. 13. Each trace (1-4) gives the average signal waveforms recorded on seven electrodes for the corresponding cluster (1-4) shown in Fig. 12. The seed electrode is 1 , on which a spike has been detected, and electrodes 2-7 are the nearest neighbors (as displayed in Fig. 10). The amplitude scale for each trace is the same as in Fig. 11.

"contaminated" neuron candidates. The ACF is a histogram of the time difference for all pairs of spikes associated with a given cluster. When a neuron spikes, there is a deadtime ("refractory period") of $\sim 1.5 \mathrm{~ms}$ in duration during which the neuron cannot fire again. Therefore, an entry in the ACF in this time difference interval is an indication of a contaminated neuron candidate. (Normally, the ACF time difference interval between $0-0.5 \mathrm{~ms}$ is not used, as a small spike sitting on top of the main spike, as the main spike amplitude drops below threshold, can appear as two spikes very close together.) The ACFs indicate that cluster 4 , with a large number of entries in the refractory period, is due to two or more neurons.

In the last step, duplicate neuron candidates are identified. Duplicate neurons will arise when the same neuron is found on two or more seed electrodes. The identification of duplicates is based on time coincidences for the spike train associated with one neuron candidate and the spike train associated with a second neuron candidate.

\section{ELECTROPHYSIOLOGICAL IMAGING}

One novel use of the multielectrode array system is to image each identified neuron in terms of the average analog waveform generated on each electrode as the neuron spikes. Fig. 14(a) shows superimposed example images of four neurons located at different locations on the electrode array, with data taken from monkey retina. For each neuron, the average electrode signal is represented by a circle at the electrode location, with a diameter given by the maximum (negative) signal amplitude. The large circles give the approximate locations of the cell bodies on the array, and the lines emanating from the cell body regions are images of the corresponding axons. Note that the axons run in parallel directions; this will help determine the orientation and location of the retinal sample with respect to the whole retina.

More detailed information can be seen in the waveforms shown on the right. Fig. 14(b) shows the bipolar signal generated at the cell body; Fig. 14(c) shows the bipolar signal of opposite polarity generated most likely by a section of the dendritic tree. Fig. 14(d)-(f) shows the triphasic axonal signals at three locations along the axon. The displacements in time, taking account of the electrode positions, give the signal propagation velocity as $1.6 \mathrm{~m} / \mathrm{s}$.

A wealth of information is thus available that we expect will contribute to neuron identification and classification. One future goal is to connect neuron classification based on visual response and physiological properties with neuron classification based on cellular anatomy. We hope to achieve this by matching the electrophysiological images taken with the multielectrode array with anatomical images taken with fluorescent microscopy. 


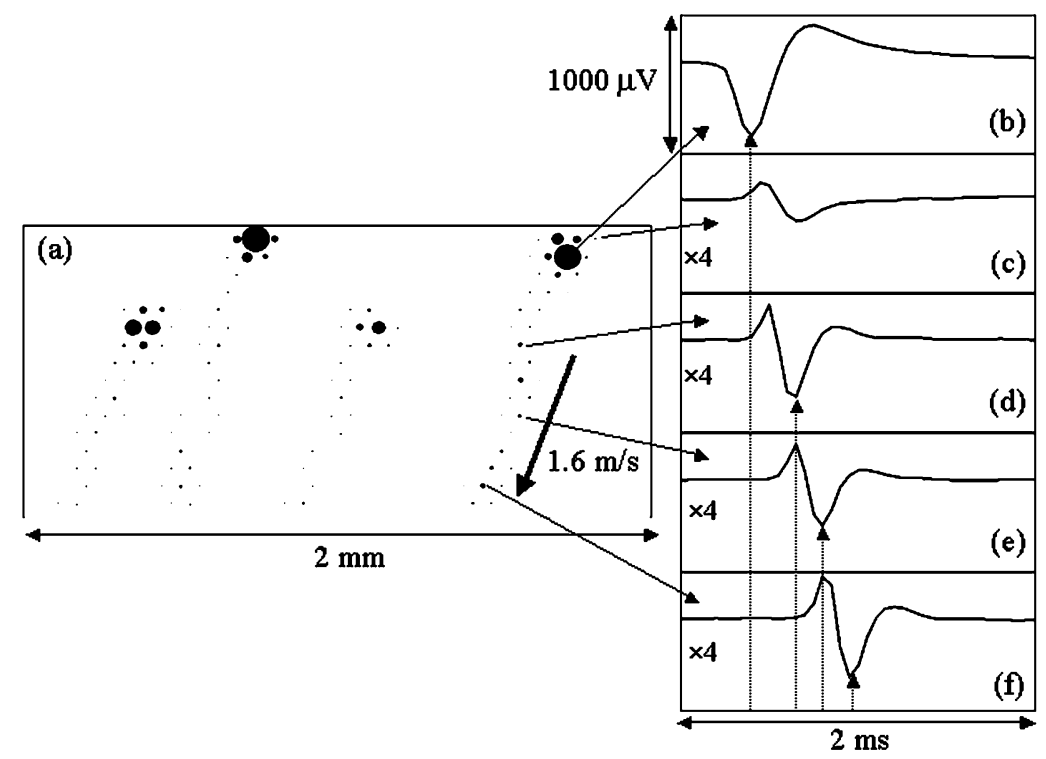

Fig. 14. (a) The superimposed electrophysiological images of four neurons. The circle diameter is proportional to the maximum (negative) signal amplitude. The cell bodies and their associated axons can be seen. (b)-(f) Selected average signal waveforms for the rightmost neuron, as recorded on the electrodes indicated by the arrows. The full vertical scale for (b) is $1000 \mu \mathrm{V}$ and $250 \mu \mathrm{V}$ for (c)-(f).

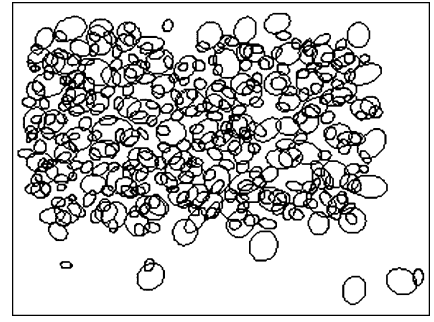

Fig. 15. Receptive fields for 364 identified neurons. Each receptive field is represented by a one-sigma contour ellipse. The rectangle has dimensions $3.2 \times$ $1.6 \mathrm{~mm}^{2}$.

\section{SOME FIRST RESUlTS ON RETINAL ORGANIZATION}

We measured the response properties of the identified neurons by correlating the visual images focused on the retina with the spiking activity of the neuron. One dynamic image used in these studies is a "white noise" image, described in [10] and [11]. Based on the white noise analysis technique, the lightsensitive region (the receptive field, RF) associated with each neuron can be measured.

Fig. 15 shows a plot of the receptive fields for 364 identified neurons in monkey retina. The RF for each neuron is based on a fit to a 2-D Gaussian; it is represented on the plot by the onesigma contour ellipse. No retinal organization is apparent from this figure.

If, however, neurons are separated into classes based on the size of their receptive fields (large or small) and their temporal response properties (ON cells detect a transition from dark to bright; OFF cells detect a transition from bright to dark), then an independent tiling of the visual region by the RGCs in each of four classes (ON-large, OFF-large, ON-small, and OFF-small) becomes apparent (Fig. 16). (Most likely, these are the ON-parasol, OFF-parasol, ON-midget, and OFF-midget RGC classes, using the terminology of neurobiology.) Note: the sparseness of the OFFsmall mosaic is likely due to inefficient detection of these small

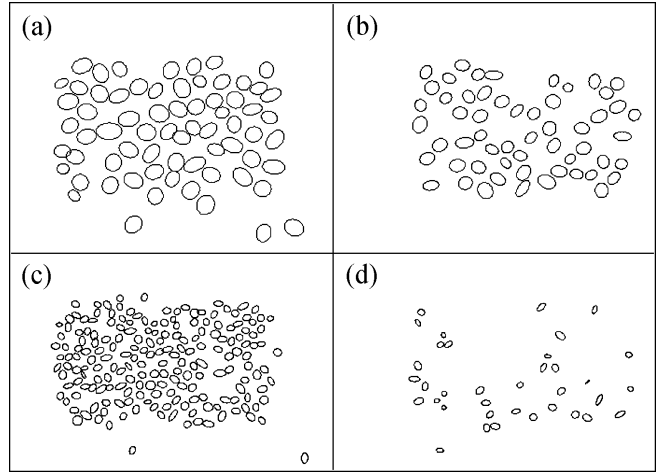

Fig. 16. Receptive fields for the same neurons as shown in Fig. 15, but plotted separately for each of four classes: (a) ON-large, (b) OFF-large, (c) ON-small, and (d) OFF-small. Each rectangle has dimensions $3.2 \times 1.6 \mathrm{~mm}^{2}$.

neurons by an electrode array with $60-\mu \mathrm{m}$ electrode spacing. This, in part, is the motivation to fabricate higher density arrays, with a $30-\mu \mathrm{m}$ electrode spacing, as mentioned in Section III.

In addition to the data shown on monkey retina mosaics, studies are underway on the organization of the guinea pig retina [11] and on receptive field microstructure [12]. We are also studying retinal response to dynamic images, such as drifting sinusoidal gratings and moving bars.

\section{SUMMARY}

We summarize the main points as follows.

- We have developed a multielectrode array system for the large-scale recording and analysis of retinal ganglion cell activity.

- Experimental data from isolated guinea pig and monkey retinas were obtained and analyzed.

- This system makes it possible, for the first time, to study visual processing and encoding by the retina in terms of 
the correlated activity of hundreds of neurons in a single preparation.

- Potential additional applications include the study of slices of brain tissue and of networks of cultured neurons.

\section{REFERENCES}

[1] A. M. Litke, "The retinal readout system: an application of microstrip detector technology to neurobiology," Nucl. Instrum. Methods A, vol. 418, pp. 203-209, 1998.

[2] — "The retinal readout system: a status report," Nucl. Instrum. Methods A, vol. 435, pp. 242-249, 1999.

[3] A. M. Litke, E. J. Chichilnisky, W. Dabrowski, A. A. Grillo, P. Grybos, S. Kachiguine, M. Rahman, and G. Taylor, "Large-scale imaging of retinal output activity," Nucl. Instrum. Methods A, vol. 501, pp. 298-307, 2003.

[4] M. Meister, J. Pine, and D. A. Baylor, "Multi-neuronal signals from the retina: acquisition and analysis," J. Neurosci. Methods, vol. 51, pp. 95-106, 1994.

[5] K. Mathieson, W. Cunningham, M. Horn, V. O'Shea, K. M. Smith, M. Rahman, A. Litke, S. Kachiguine, and E. J. Chichilnisky, "Large-area microelectrode arrays for recording of neural signals," presented at the IEEE NSS-MIC 2003 Conf. Rec., N30-5.
[6] W. Dabrowski, P. Grybos, P. Hottowy, A. Skoczen, K. Swientek, N. Bezayiff, A. A. Grillo, S. Kachiguine, A. M. Litke, and A. Sher, "Development of integrated circuits for readout of microelectrode arrays to image neuronal activity in live retinal tissue," presented at the IEEE NSS-MIC 2003 Conf. Rec., N30-6.

[7] LabVIEW Software From National Instruments, Austin, TX. [Online] Available: http://www.ni.com/labview/

[8] Multifunction I/O Board PCI-6110E From National Instruments, Austin, TX. [Online] Available: http://www.ni.com/

[9] Pattern Generation Board PCI-6534 From National Instruments, Austin, TX. [Online] Available: http://www.ni.com

[10] H. M. Sakai, "White-noise analysis in neurophysiology," Physiol. Rev., vol. 72, pp. 491-505, 1992.

[11] E. J. Chichilnisky, "A simple white noise analysis of neuronal light responses," Network: Comput. Neural Systems, vol. 12, pp. 199-213, 2001.

[12] M. I. Grivich, D. Petrusca, R. S. Kalmar, A. Sher, E. J. Chichilnisky, and A. M. Litke, "Classification of guinea pig retinal ganglion cells using large scale multielectrode recordings," in Annu. Meeting Society for Neuroscience 2003.

[13] R. S. Kalmar, A. Sher, M. I. Grivich, D. Petrusca, A. M. Litke, and E. J. Chichilnisky, "Receptive field microstructure of mammalian retinal ganglion cells revealed by large scale multielectrode recording," in Annu. Meeting Society for Neuroscience 2003. 